\title{
Towards the elimination of dog-mediated rabies: development and application of an evidence-based management tool
}

Kristyna Rysava ${ }^{1,2^{*}}$ D, Tamara Mancero ${ }^{3}$, Eduardo Caldas ${ }^{4}$, Mary Freire de Carvalho ${ }^{3}$, André P. B. Castro ${ }^{5}$, Veronica Gutiérrez ${ }^{6}$, Daniel T. Haydon², Paul C. D. Johnson², Rebecca Mancy², Lúcia R. Montebello", Silene M. Rocha ${ }^{5}$, Jesús F. Gonzalez Roldan ${ }^{6}$, Marco Antonio Natal Vigilato ${ }^{3}$, Victor Del Rio Vilas ${ }^{3,7}$ and Katie Hampson ${ }^{2}$

\begin{abstract}
Background: International organizations advocate for the elimination of dog-mediated rabies, but there is only limited guidance on interpreting surveillance data for managing elimination programmes. With the regional programme in Latin America approaching elimination of dog-mediated rabies, we aimed to develop a tool to evaluate the programme's performance and generate locally-tailored rabies control programme management guidance to overcome remaining obstacles.
\end{abstract}

Methods: We developed and validated a robust algorithm to classify progress towards rabies elimination within sub-national administrative units, which we applied to surveillance data from Brazil and Mexico. The method combines criteria that are easy to understand, including logistic regression analysis of case detection time series, assessment of rabies virus variants, and of incursion risk. Subjecting the algorithm to robustness testing, we further employed simulated data sub-sampled at differing levels of case detection to assess the algorithm's performance and sensitivity to surveillance quality.

Results: Our tool demonstrated clear epidemiological transitions in Mexico and Brazil: most states progressed rapidly towards elimination, but a few regressed due to incursions and control lapses. In 2015, dog-mediated rabies continued to circulate in the poorest states, with foci remaining in only 1 of 32 states in Mexico, and 2 of 27 in Brazil, posing incursion risks to the wider region. The classification tool was robust in determining epidemiological status irrespective of most levels of surveillance quality. In endemic settings, surveillance would need to detect less than $2.5 \%$ of all circulating cases to result in misclassification, whereas in settings where incursions become the main source of cases the threshold detection level for correct classification should not be less than 5\%.

(Continued on next page)

\footnotetext{
* Correspondence: K.Rysava@warwick.ac.uk

${ }^{1}$ University of Warwick, School of Life Sciences, Gibbet Hill Road, Coventry, UK

${ }^{2}$ University of Glasgow, Institute of Biodiversity, Animal Health and Comparative Medicine, Graham Kerr building, MVLS, Glasgow G12 8QQ, UK Full list of author information is available at the end of the article
}

(c) The Author(s). 2020 Open Access This article is licensed under a Creative Commons Attribution 4.0 International License, which permits use, sharing, adaptation, distribution and reproduction in any medium or format, as long as you give appropriate credit to the original author(s) and the source, provide a link to the Creative Commons licence, and indicate if changes were made. The images or other third party material in this article are included in the article's Creative Commons licence, unless indicated otherwise in a credit line to the material. If material is not included in the article's Creative Commons licence and your intended use is not permitted by statutory regulation or exceeds the permitted use, you will need to obtain permission directly from the copyright holder. To view a copy of this licence, visit http://creativecommons.org/licenses/by/4.0/. The Creative Commons Public Domain Dedication waiver (http://creativecommons.org/publicdomain/zero/1.0/) applies to the data made available in this article, unless otherwise stated in a credit line to the data. 


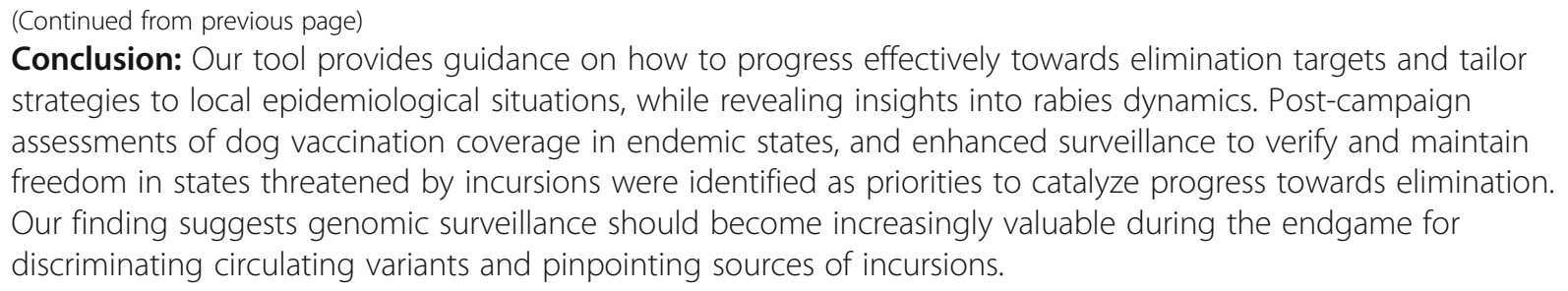

Keywords: Canine rabies, Decision support tool, Freedom from disease, Interruption of transmission, Management recommendations, Mass dog vaccination, Scientific guidance, Surveillance

\section{Summary}

International agencies have committed to the goal of global elimination of dog-mediated rabies. Considerable progress towards eliminating dog-mediated rabies has been achieved in Latin America; however, the region has encountered setbacks and further work is still needed to achieve this goal. Scientific guidance for managing rabies control programmes to ensure that setbacks are overcome and that progress continues during the endgame is critical, yet often limited and lacking in specific recommendations. Characteristic patterns of disease dynamics are indicative of progress towards elimination, and through their identification, tailored guidance can be provided. Here, we develop a robust tool to evaluate progress from routinely collected surveillance data and to inform rabies elimination programmes of where and how surveillance and control efforts need improvement. We developed the tool using the surveillance database for rabies in Latin America (SIRVERA) maintained by the Pan American Health Organization. We demonstrate the utility of this tool to support policymakers and rabies programme managers at regional, national and subnational levels through its application in Mexico and Brazil. We further developed an interactive web interface for communicating this progress and guidance (https:// boydorr.shinyapps.io/paho_rabies/), which can be applied throughout Latin America and other regions around the world as regional rabies elimination programmes mature using their established surveillance systems. Priorities highlighted by applying this tool in Mexico and Brazil were to: strengthen the delivery and monitoring of dog vaccination campaigns in identified persistent foci; and to enhance surveillance to distinguish virus variants, to support rapid response to incursions and to verify disease freedom.

\section{Motivation}

Rabies has been eliminated from domestic dog populations in high-income countries, but remains a major public health concern in low- and middle-income countries. Every year, thousands of people die and billions of dollars are lost due to rabies spread by domestic dogs
[1]. Regional and national targets for the elimination of dog-mediated rabies have now been set [2] and control programmes are underway around the world [3-5]. A suite of resources is available to support countries in the global campaign to eliminate human deaths from dog mediated-rabies by 2030 [6], spanning the entire process from zoonotic disease prioritization [7, 8], calculating programmatic resource needs [9] to validation of zero human deaths and verification of interruption of transmission and rabies freedom [10, 11]. Many of these tools are accessible from the Rabies Blueprint Platform (www. rabiesblueprint.org), a live document hosting up-to-date and comprehensive case studies, procedures and protocols for rabies control and prevention [12]. These complementary tools can be used strategically to foster sustainable collaborations for rabies elimination [13]. In particular, the Stepwise Approach towards Rabies Elimination (SARE) is for use by countries to self-assess their national programmes and should be repeated periodically to benchmark progress and revise priorities [14]. However, for countries or regions with already wellestablished rabies control programmes including surveillance systems generating data on programme impacts, there is limited guidance on how to interpret those data and tailor activities accordingly to ensure progress towards elimination remains on track.

\section{Rabies management in Latin America}

Most progress has been made towards the regional elimination of dog-mediated rabies in Latin America. Since 1983, national dog vaccination programmes coordinated by the Pan American Health Organisation (PAHO) have controlled canine rabies across much of the Western Hemisphere, reducing incidence by over 99\% [3]. But, as the region approaches elimination, differences in progress have emerged [15]. Variability in the implementation of control measures, as well as geographic, population and socioeconomic differences likely underpin differential progress in rabies control. However, observed patterns may also reflect variation in the quality of surveillance. Clarifying the relationship between these influences and their effects on rabies detection and 
circulation is key to designing effective interventions during the endgame. For example, some areas have ostensibly achieved rabies freedom and now face competing priorities that create pressure to reduce expenditure on rabies control. In contrast, other areas are struggling to control rabies, and continued circulation poses a risk for reintroduction into neighbouring areas, potentially threatening the success of the regional programme [16, 17]. Consequently, there is an increasingly urgent need to address these differences within the region and for targeted scientific guidance to ensure continued progress. By tailoring efforts to local epidemiological situations, it should be possible to accelerate progress towards elimination and sustainable freedom from disease.

\section{Management tool}

We present a tool for programme managers and practitioners working at regional, national and subnational levels to guide their programme management using routine surveillance data from their local area and from neighbouring areas that may influence their epidemiological situation. The tool was designed using data from SIRVERA, a regional rabies surveillance database first established in 1969 (http://sirvera.panaftosa.org.br/). As such, the tool is targeted towards countries or regions with already well-established rabies control programmes and surveillance systems with regular submission of samples from suspect animals. Our principles in developing this tool were that: (1) it should be possible to classify the epidemiological situation objectively in defined geographic areas using routine surveillance data; (2) categories and their criteria should be easy to understand; (3) classification should provide insights into the effectiveness of current management and guidance for further progression, including readiness to undertake independent verification of rabies freedom [10, 11]. Here we describe the rationale for the tool, its methodological development and assessment of its robustness. We apply this tool sub-nationally across Mexico and Brazil, where dog rabies control programmes have been ongoing since the 1980s. Using the countries' routine surveillance data, we reveal historical and current patterns of rabies circulation, and generate guidance for surveillance and control strategies that are tailored to specific localities to facilitate progress towards elimination.

\section{Methods}

\section{Algorithm development}

Our aim was to develop a tool to help rabies managers and practitioners to understand progress towards rabies elimination by distinguishing areas with ongoing transmission from areas where efforts have controlled rabies and have potentially interrupted transmission. To be both useful for programme managers and epidemiologically meaningful, we envisaged a tool for application across relatively large administrative units such as states, provinces or districts, rather than smaller units such as villages or municipalities. We focused on case reports (i.e. numbers of laboratory confirmed cases per unit area and per unit time), as this is the simplest information recorded in most surveillance systems for rabies. In addition, we also identified scenarios where viral characterization, specifically identification of the viral variant of the detected case, provides additional clarity. We developed a classification algorithm to evaluate and distinguish characteristic patterns when applied to extended periods (minimum of 5 years) of complete monthly surveillance data. In the process of refining the classification algorithm through application to states (major sub-national administrative units) in Brazil and Mexico, we discussed the resultant classifications with state-level and national stakeholders to clarify our interpretation.

\section{Data}

For development of the algorithm we used data on laboratory confirmed rabies cases in dogs from Mexico and Brazil submitted to SIRVERA between January 2005 and December 2015. The raw data are publicly available from the SIRVERA website or can be shared on request from sirvera@paho.org. Both countries have ongoing rabies control programmes that were initiated in the 1980s, with annual dog vaccination campaigns managed at the state-level, and major declines in laboratory confirmed rabies cases recorded since the start of their programmes [18]. These countries were selected by PAHO due to the quality of their surveillance, which was considered adequate for this classification both at national and state level. This qualitative assessment was based on previously recorded declines in incidence, continued detection of wildlife rabies variants and submission of samples, lack of reported human rabies deaths from canine rabies, diagnostic laboratory proficiency and completed monthly records in SIRVERA. SIRVERA has recently been updated to capture information on virus variants. However, since this information had not been routinely recorded in SIRVERA previously, data on virus variants were provided separately by the Ministries of Health in Mexico and Brazil.

\section{Classification criteria}

We developed the algorithm to classify second-level administrative units (hereafter referred to as states) into 5 putative categories: Endemic, Declining, Intermittent, $A b$ sent-Vulnerable and Absent (Table 1), based on characteristic patterns identified via a set of objective criteria (Fig. 1, Table 2). We caution that classification to the 
Table 1 Putative epidemiological classifications

1) ENDEMIC TRANSMISSION: Canine rabies (variants 1 \& 2 in Latin America [19]) detected over at least two consecutive months during the previous 2 years, indicating focal transmission. No significant decrease in the frequency of months with case detection over the previous 5 years. 2) DECLINING TRANSMISSION: At least 1 month with detected canine rabies cases in the previous 2 years, but a declining frequency of months with detected cases over the previous 5 years.

3) INTERMITTENT DETECTION: Canine rabies cases detected during the past 2 years but not over consecutive months. No temporal trend in the frequency of months with detected cases during the previous 5 years.

4) ABSENT-VULNERABLE: Either: (i) canine rabies cases not detected in the previous 2 years, but neighbouring an area where rabies is Endemic or Declining and therefore vulnerable to incursions; or (ii) a single month with cases detected during the previous 2 years, but no case detection prior to that month for at least 2 years (i.e. recently experienced an incursion that did not lead to further spread).

5) ABSENT: No cases of canine rabies cases detected during the last 2 years and minimal risk of incursion (i.e. not neighbouring with any Endemic or Declining states). categories Absent-Vulnerable and Absent does not satisfy OIE recommendations for declaration of freedom. The classification criteria and their rationale are outlined below.

\section{Case detection}

For each state, we calculated the time since the last detected case, and categorized case detection in the last 2 years as: present in at least two consecutive calendar months; present, but not over consecutive months; and absent (Criterion I). This criterion was used together with trends in case detection (Criterion II) to distinguish between Endemic, Declining and Intermittent classifications. States where rabies was detected in a single month during the last two years but that had an absence of cases prior to that month for at least 2 years were classified as putatively Absent-Vulnerable. Among states with no case detection in the previous 2 years, we distinguished between two categories (Absent and Absent-Vulnerable) according to incursion risk (Criterion IV).

The rationale for Criterion I was based on the following arguments. Firstly, following an introduction, the serial interval for rabies means that $\sim 35 \%$ of secondary

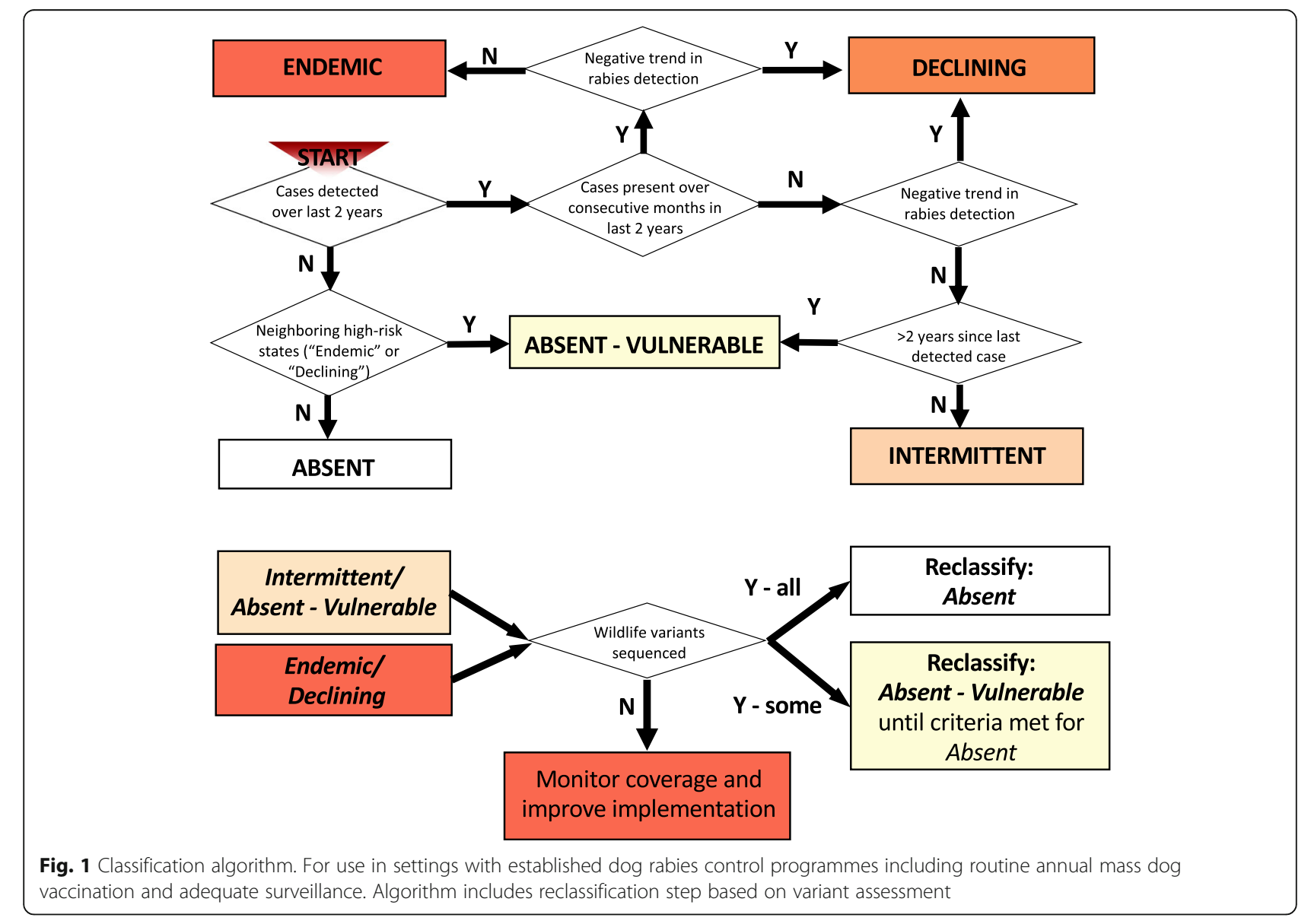


Table 2 Classification criteria (corresponding to Fig. 1). NA - not applicable, V1 and V2 - canine rabies genetic variants of type 1 and 2 respectively in Latin America [19]

\begin{tabular}{|c|c|c|c|c|}
\hline Classification & Cases in last 2 years & $\begin{array}{l}\text { Trend (model } \\
\text { coefficient) over } 5 \text { years }\end{array}$ & $\begin{array}{l}\text { Absence (> } 2 \text { yrs with no V1 } \\
\text { or V2) }\end{array}$ & Incursion risk \\
\hline Endemic & $\begin{array}{l}\text { Yes, V1 \& V2, over at least two } \\
\text { consecutive months }\end{array}$ & None/ positive & NA & NA \\
\hline Declining & Yes, V1 \&/V2 & Negative & NA & NA \\
\hline Intermittent & $\begin{array}{l}\text { Yes, but not over two or more } \\
\text { consecutive months }\end{array}$ & None/ positive & NA & NA \\
\hline $\begin{array}{l}\text { Absent - } \\
\text { Vulnerable (i) or } \\
\text { (ii) }\end{array}$ & $\begin{array}{l}\text { (i) No } \\
\text { (ii) V1 \&N2 in } 1 \text { month only }\end{array}$ & (i \& ii) NA & $\begin{array}{l}\text { (i) At least last } 2 \text { years } \\
\text { (ii) }>2 \text { yr absence prior to last } \\
\text { detected case(s) }\end{array}$ & $\begin{array}{l}\text { (i \& ii) Adjacent to Endemic or } \\
\text { Declining area(s) }\end{array}$ \\
\hline Absent & No & NA & NA & $\begin{array}{l}\text { Not adjacent to any Endemic } \\
\text { or Declining areas }\end{array}$ \\
\hline
\end{tabular}

cases are expected to occur within 1 month. A lack of detection over consecutive months therefore suggests secondary transmission is not sustained and no other introductions occurred. A lack of detection could also result from inadequate surveillance, but in combination with temporal trends in case detection (Criterion II), this would result in an Intermittent classification. Our decision to use a two-year window for the classification of Absent is based upon modelling work showing that while mass dog vaccinations are ongoing, a two-year period without detection of rabies, should be sufficient to be confident of elimination, even at realistically low levels of surveillance [20].

\section{Temporal trends in case detection}

We used monthly state-level time series of laboratory confirmed cases spanning several years of surveillance data to assess temporal trends. After converting these time series to monthly state-level binary data (presenceabsence), we fitted a logistic regression to determine whether the monthly probability of case detection was increasing, decreasing or showed no trend (Supplementary Figures S1). Using binary data in this way (as opposed to case counts) aims to overcome spurious inference from either fluctuation in incidence or detection that may reflect awareness, investment or other external drivers (see section on Algorithm Testing and Validation, and Supplementary Figures S1 and S2). Since cases are detected during the month when rabid animals become infectious, but infection could also be present prior to this due to latent infections we adjusted the time series for our regressions to include the month prior to case detection as presence. If cases were detected over at least two consecutive calendar months (excluding the latent period) in the past 2 years, we classified states with time series exhibiting significant declines in case detection as Declining, versus those with no trend or an increasing frequency of months with detected cases as Endemic. For states where cases were detected in the past 2 years but not over consecutive months (excluding the latent period), we classified the states as either Declining or Intermittent depending upon whether the data exhibited decreasing or no temporal trend in detection. It should be noted that for the classification of Declining to apply, we required that a state be implementing annual (or more frequent) dog vaccination campaigns.

\section{Variant identification}

Cases detected in the previous 2 years were assessed to determine whether they were due to variants associated with dogs (V1 \& V2) or with other species (e.g. bats, terrestrial wildlife). Classifications were then updated with wildlife-associated variants removed. A reclassification to Absent or Absent-Vulnerable could therefore occur if all detected cases from the last 2 years were due to wildlife variants. This criterion provides a check as to whether a sylvatic virus may have spilled over into domestic dogs and whether further investigation is required to understand complexities in transmission and maintenance.

\section{Incursion risk}

We assessed the risk of incursions into non-endemic Absent states based on shared borders with high-risk states or countries (Endemic or Declining). We classified as Absent-Vulnerable those states with at least one neighbouring state classified as either Endemic or Declining (Table 2).

\section{Algorithm testing and validation}

We subjected our classification algorithm to robustness testing and validation. Initially we applied our algorithm to SIRVERA data from 2005 to 2015 for Mexico and Brazil, classifying states in both countries retrospectively using a rolling multi-year time window. To explore how the length of the time series affected the sensitivity of the logistic regression approach to identify trends, we refitted to incrementally truncated rolling windows of 
presence-absence data (from 2 to 10 years), and compared classifications according to how many years of data were included. For temporal trends, we explored the possibility of modelling incidence (case counts) using Poisson regression rather than Logistic regression (presence-absence timeseries) (Figure S1). We also considered whether missing information (major and minor administrative units corresponding to states and municipalities respectively) would indicate inadequate surveillance and the impact of missing information on classifications in Mexico and Brazil.

To assess how well our algorithm performed under different levels of surveillance and/or reporting quality, we simulated canine rabies dynamics, and then resampled our simulated time series to mimic differing levels of surveillance before applying our classification algorithm.

To simulate canine rabies, we adapted an existing model [21]. Our aim was to simulate epidemiological dynamics not specific to a particular setting, but for populations of a similar size and scale to those in states in Brazil and Mexico to test the algorithm performance. We therefore created populations equivalent in size to the average dog population of states in Mexico (mean 634,361 , median 409,877, simulated population 505,341), sufficient to support rabies persistence without incursions. Epidemiological parameter estimates were derived from data from Tanzania on rabid dog incubation and infection periods, and movement and biting distributions [22], that are expected to be broadly similar irrespective of the geographical setting. We modelled dog demography explicitly, tracking the number of dogs in each spatially-defined area, so that we could simulate vaccination coverage and its waning with population turnover. We heterogeneously distributed dog populations across $1 \mathrm{~km}^{2}$ grid cells by scaling up georeferenced dog population data from Tanzania. The purpose of this was to capture reasonable population clustering known to affect rabies persistence, in the absence of detailed spatial data on dog distributions from Latin America. We assumed high dog population turnover (average lifespan of 2.5 years), which is representative of the short lifeexpectancy of dogs in low socio-economic settings with endemic rabies [23], and similar to those reported for populations with endemic rabies in Brazil and Mexico $[24,25]$. We have previously found this model to be applicable in different settings $[20,21,26]$ and we checked for appropriate dynamics (incidence patterns and persistence) prior to applying the classification algorithm.

We initialized simulations, with an average incidence of around $1 \%$ of the dog population per annum [27], with 450 cases distributed across the landscape with probability proportional to dog density. We then generated 10 stochastic realizations of 10 years of simulated cases for the following scenarios: (a) with no control measures in place, (b) with mass dog vaccination campaigns achieving an average of $60 \%$ coverage with realistic spatial heterogeneity, (c) with reduced vaccination coverage to $20 \%$ and (d) $10 \%$. We also considered (e) incursions approximately every 6 months under high dog vaccination coverage (scenario $b$ ) and (f) reduced vaccination coverage (scenario $\mathrm{c}$ ) and (g) into a rabies-free population. This set of $10 \times 7$ simulation runs each captured the full spectrum of classifications (Table 1). We then resampled the simulated time series to mimic differing levels of surveillance (i.e. 1, 2, 5, 10, 20\% of circulating cases detected by the surveillance system) and applied our classification algorithm to each resampled dataset to assess the algorithm robustness to surveillance quality.

\section{Algorithm application}

We applied our final algorithm to data from Mexico and Brazil from 2005 until 2015 in order to assess transitions from one classification to another over this period. We report these classifications (see Table 1) and their epidemiological interpretation and implications for management. We also developed a Shiny application as a dynamic interface for exploring the classification of states in Mexico and Brazil over this time period and the interpretation in terms of rabies control programme management.

\section{Results}

\section{Algorithm development}

On the basis of the rationale described in the methods we developed a two-stage classification process for use in settings with established dog rabies control programmes with annual or more frequent mass dog vaccination campaigns at second-level administrative units and adequate surveillance systems in place i.e. at least at Stage 3 within the SARE [28]. Our final algorithm is described in Fig. 1 and Table 2, with the temporal trend in case detection criterion applied over 5 years of monthly surveillance data.

\section{Algorithm testing and validation}

During the algorithm development and testing we found that time series of confirmed rabies cases were highly variable (Table S1, Figures S1 and S2). Overall, we found that presence-absence time series and their trends upon logistic regression were more informative and less sensitive to biases due to population sizes or fluctuations in reporting than time series of case counts (Table S1, Figures S1 and S2 insets). Generally 4-6 years appeared to be a sufficient and useful time window to detect consistent temporal trends in case detection and be responsive to changing dynamics (Figure S3, S4 and boydorr. 
shinyapps.io/paho_rabies/). Using shorter time windows (<4 years) magnified transient patterns, whereas dynamical transitions were less apparent with longer windows (>6 years). We therefore suggest the trend in case detection criterion be applied over a 5-year time window.

Missing information is a prevalent problem in routine surveillance, potentially misleading conclusions regarding the occurrence and severity of disease circulation. We examined the effect of missing location information (major and minor administrative units corresponding to states and municipalities respectively) on classifications. Between 2005 and 2015, of 442 cases reported in Mexico 386 had no municipality information reported and 34 had no state information. Of 558 cases reported in Brazil, 326 had no municipality information reported and 14 cases had no state information. However, from 2011 onwards, municipalities and states were reported for all cases in both countries. Missing major administrative level information for cases (2.5\% in Brazil, $7.6 \%$ in Mexico) did not affect the 2015 classification, but may have had a small impact in earlier years. Nonetheless the epidemiological interpretation of transitions was clear (see Algorithm Application).

Through subsampling simulated time series, we confirmed that our classification tool was robust in determining epidemiological status. Surveillance had to reach very low levels or be sufficiently biased for states to be misclassified (Fig. 2). In endemic settings (Endemic/ Declining), surveillance would need to detect less than $2.5 \%$ of all circulating cases to result in misclassification. This threshold detection level for correct classification, however, increased to $5 \%$ as incursions become the main source of cases i.e. for settings classified as Intermittent or Absent-Vulnerable.

\section{Algorithm application}

Classifications of Brazilian and Mexican states in 2005, 2010 and 2015 are shown in Fig. 3 and transitions over this time period by month can be viewed via the Shiny application: https://boydorr.shinyapps.io/paho_rabies/ (and in Figures S3 and S4). Over this decade, considerable progress was evident in both countries, with many Endemic, Declining or Intermittent states transitioning to Absent or Absent-Vulnerable. In 2015, most states in both countries were classified as either Absent or $A b$ sent-Vulnerable (Table 3). Focal circulation persisted only in southern Mexico and northeast Brazil, except for an outbreak in Mato Grosso do Sul, Brazil in 2015 that originated from a transboundary incursion and that was rapidly controlled.

In Mexico, only Chiapas state on the border with Guatemala was classified as Endemic in 2015 (Fig. 3). Yucatán state was classified as Intermittent rather than Declining, because one of the two detected cases in the prior 2 years (2014) was a wildlife variant. Incursion risks generally declined as rabies was controlled in central Mexico, primarily in Mexico City and surrounding states of Puebla and Veracruz (Fig. 3), but circulation in Chiapas and neighbouring Guatemala still put other states at risk of re-emergence (Fig. 3a, b). Yucatán is one of the few states that switched from a putatively Absent classification back to Endemic indicating that investigation, possibly into wildlife circulation is warranted (Figure S3). A prolonged absence in Chiapas led to its reclassification from Endemic to Absent-Vulnerable in 2006-2007 prior to detection of rabies again in 2008 and reversion to Endemic (Figure S3). It is unclear whether these transitions in Chiapas were the result of improved surveillance or incursions from Guatemala.

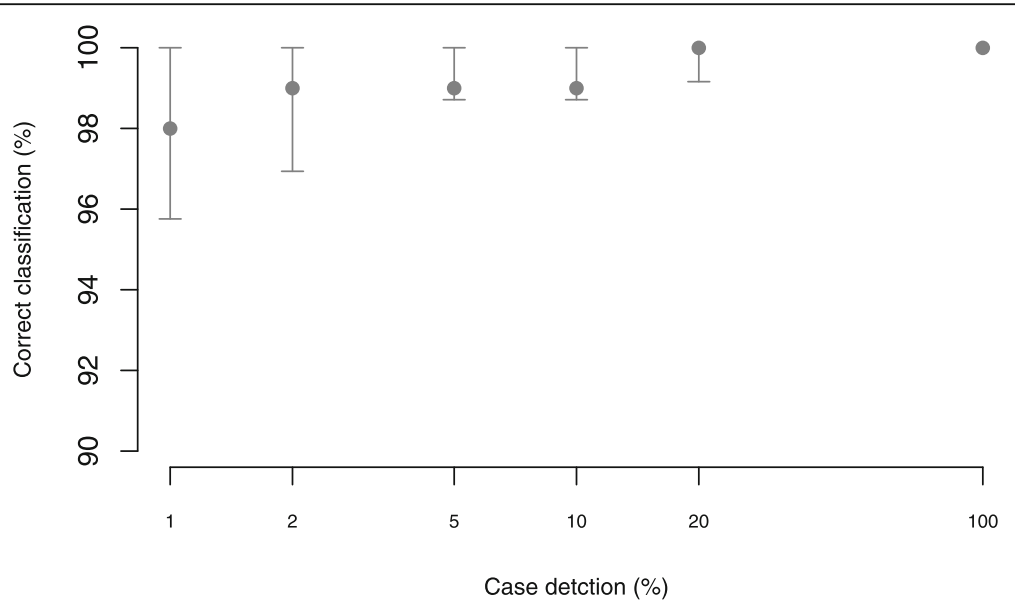

Fig. 2 Performance of the classification algorithm according to the quality of surveillance. Note that case detection is plotted on a log scale and that the $y$-axis is shown from 90 to $100 \%$. Percentage of circulating cases detected (case detection) measures surveillance quality and here relates to the extent to which suspect rabid animals are investigated thereby enabling sample collection and subsequent laboratory testing 


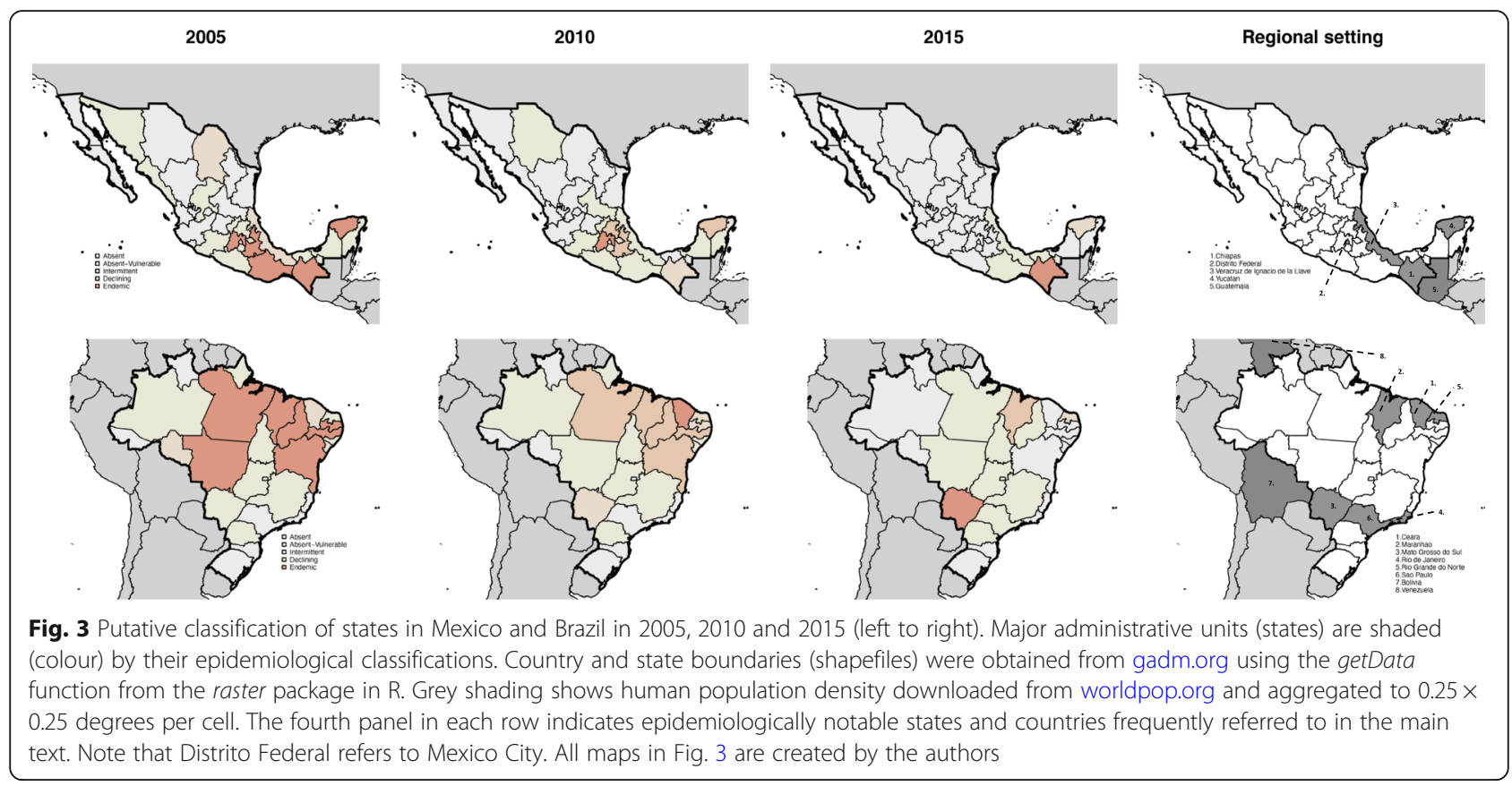

Missing data on locations of cases during this period may have affected the earlier classifications.

In 2015 in Brazil, only Mato Grosso do Sul was classified as Endemic as a consequence of the outbreak in late 2015, while in Northeast Brazil, Maranhão and Rio Grande do Norte were classified as Declining and Intermittent respectively. Incursion risks are largely driven by rabies circulation in Northeastern states where until recently dog rabies variants co-circulated together with wildlife variants (predominantly in Rio Grande de Norte, Ceará and Sergipe); however, continued dog rabies control efforts appear to have reduced circulation to just Maranhão and possibly Rio Grande de Norte. The outbreak in Mato Grosso do Sul was detected in Corumba, a border town with Bolivia, and was restricted to the municipalities of Corumba and Ladario (Fig. 3). São Paulo State was initially classified as Intermittent, but variant information indicated that recent cases were associated with wildlife variants rather than canine rabies (V1 or V2).

We derived management guidance for each classification, summarized in Fig. 4. The Shiny app that we developed also visualizes progress towards elimination across the region, and was designed to communicate tailored guidance on rabies management at the state level: https://boydorr.shinyapps.io/paho_rabies/.

\section{Discussion}

\section{Key findings}

Guidance for established rabies elimination programmes, such as those in Latin America, is relatively limited and not geographically specific $[10,29]$. The management tool that we have developed and validated using surveillance data from Mexico and Brazil allows to identify mutually exclusive epidemiological situations from a simple algorithm, without the need for extensive statistical expertise (illustrated at: https://boydorr.shinyapps.io/paho rabies/). By classifying states in these countries, we determined surveillance and control priorities at local, national and regional levels, and derived tailored guidance on how to continue progressing towards elimination, while revealing insights into rabies dynamics (Figs. 3 and 4, Table 4).

Progress towards elimination was evident in both Mexico and Brazil; however, focal transmission remains a threat for re-emergence in ostensibly rabies-free states. Connectivity has been demonstrated to play a critical role in rabies persistence, with infection maintained across, and driven by, large interconnected metapopulations (rather than dense conurbations) [30, 31]. Our classification identified metapopulations that support focal transmission, such as in central Mexico in the recent past (e.g. in 2010, Fig. 3), and the cross-border area between Mexico and Guatemala, as well as in Northeast Brazil (Fig. 3). There is an urgent need to improve surveillance and control in remaining foci, including endemic bordering countries. For example, the outbreak in the municipality of Corumba, Mato Grosso do Sol, on the Brazil-Bolivia border is rumoured to have spread from Bolivia, even though no cases were reported to SIRVERA from Bolivia in over 3 years. Incursions with long-lasting consequences have been reported elsewhere in Latin America [32], and globally [20, 33], and threaten the regional elimination effort. Moreover, rabies still 
Table 3 Putative classification of states in Mexico and Brazil showing progression from 2005 to 2015. States presenting incursion risks highlighted in bold italics

\begin{tabular}{|c|c|c|c|}
\hline MEXICO & 2005 & 2010 & 2015 \\
\hline Aguascalientes & Absent & Absent & Absent \\
\hline Baja California & Absent & Absent & Absent \\
\hline Baja California Sur & Absent & Absent & Absent \\
\hline Campeche & Absent-Vulnerable & Absent-Vulnerable & Absent \\
\hline Coahuila de Zaragoza & Intermittent & Absent & Absent \\
\hline Colima & Absent & Absent & Absent \\
\hline Chiapas & Endemic & Intermittent & Endemic \\
\hline Chihuahua & Absent & Absent-Vulnerable & Absent \\
\hline Distrito Federal & Intermittent & Absent-Vulnerable & Absent \\
\hline Durango & Absent & Absent & Absent \\
\hline Guanajuato & Absent & Absent & Absent \\
\hline Guerrero & Absent-Vulnerable & Absent-Vulnerable & Absent \\
\hline Hidalgo & Absent-Vulnerable & Declining & Absent \\
\hline Jalisco & Absent & Absent & Absent \\
\hline Mexico & Endemic & Endemic & Absent \\
\hline Michoacan de Ocampo & Absent-Vulnerable & Absent-Vulnerable & Absent \\
\hline Morelos & Absent-Vulnerable & Absent-Vulnerable & Absent \\
\hline Nayarit & Absent & Absent & Absent \\
\hline Nuevo Leon & Absent & Absent & Absent \\
\hline Oaxaca & Endemic & Absent-Vulnerable & Absent-Vulnerable \\
\hline Puebla & Endemic & Declining & Absent \\
\hline Queretaro Arteaga & Absent-Vulnerable & Absent-Vulnerable & Absent \\
\hline Quintana Roo & Absent-Vulnerable & Absent-Vulnerable & Absent \\
\hline San Luis Potosi & Absent & Absent-Vulnerable & Absent \\
\hline Sinaloa & Absent-Vulnerable & Absent & Absent \\
\hline Sonora & Absent-Vulnerable & Absent & Absent \\
\hline Tabasco & Absent-Vulnerable & Absent & Absent-Vulnerable \\
\hline Tamaulipas & Absent & Absent & Absent \\
\hline Tlaxcala & Intermittent & Absent-Vulnerable & Absent \\
\hline Veracruz de Ignacio de la Llave & Intermittent & Absent-Vulnerable & Absent-Vulnerable \\
\hline Yucatan & Endemic & Declining & Intermittent \\
\hline Zacatecas & Absent-Vulnerable & Absent & Absent \\
\hline BRAZIL & 2005 & 2010 & 2015 \\
\hline Acre & Absent & Absent & Absent \\
\hline Alagoas & Absent-Vulnerable & Absent-Vulnerable & Absent \\
\hline Amapa & Absent-Vulnerable & Absent-Vulnerable & Absent \\
\hline Amazonas & Absent-Vulnerable & Absent-Vulnerable & Absent \\
\hline Bahia & Endemic & Declining & Absent \\
\hline Ceara & Intermittent & Endemic & Absent \\
\hline Distrito Federal & Absent & Absent & Absent \\
\hline Espirito Santo & Absent & Absent & Absent \\
\hline Goias & Absent-Vulnerable & Absent-Vulnerable & Absent-Vulnerable \\
\hline Maranhao & Endemic & Declining & Declining \\
\hline
\end{tabular}


Table 3 Putative classification of states in Mexico and Brazil showing progression from 2005 to 2015. States presenting incursion risks highlighted in bold italics (Continued)

\begin{tabular}{llll}
\hline MEXICO & $\mathbf{2 0 0 5}$ & $\mathbf{2 0 1 0}$ & $\mathbf{2 0 1 5}$ \\
\hline Mato Grosso & Endemic & Absent-Vulnerable & Absent-Vulnerable \\
Mato Grosso do Sul & Absent-Vulnerable & Intermittent & Endemic \\
Minas Gerais & Absent-Vulnerable & Absent-Vulnerable & Absent-Vulnerable \\
Para & Endemic & Declining & Absent-Vulnerable \\
Paraiba & Endemic & Intermittent & Absent \\
Parana & Absent-Vulnerable & Absent-Vulnerable & Absent-Vulnerable \\
Pernambuco & Endemic & Declining & Absent \\
Piaui & Endemic & Declining & Absent-Vulnerable \\
Rio de Janeiro & Absent & Absent & Absent \\
Rio Grande do Norte & Absent-Vulnerable & Intermittent & Intermittent \\
Rio Grande do Sul & Absent & Absent & Absent \\
Rondonia & Intermittent & Absent & Absent \\
Roraima & Absent & Absent & Absent \\
Santa Catarina & Absent & Absent & Absent \\
Sao Paulo & Absent & Absent & Absent-Vulnerable \\
Sergipe & Intermittent & Absent-Vulnerable & Absent \\
Tocantins & Absent-Vulnerable & Absent-Vulnerable & Aulnerable \\
\hline
\end{tabular}

persists in a few states where control programmes have been ongoing for decades. We suggest that in these Endemic states, post-vaccination monitoring is needed to identify the causes of slow progress, which likely relate to inadequacies in dog vaccination campaigns. Vaccination coverage in Latin America has generally been estimated retrospectively from human:dog ratios. However, human:dog ratios can be heterogeneous and change considerably over time [34]. Estimating numbers of distinct dogs vaccinated can also be difficult, as some dogs are vaccinated repeatedly during outbreak responses unless concerted efforts are made to target areas missed during campaigns. Gaps in coverage were previously found to be a critical factor in rabies control, prolonging progress towards elimination [20, 26]. Post-campaign assessments of coverage are needed to both identify and remedy problematic areas [35]. Strengthening the delivery and monitoring of dog vaccination campaigns in areas with focal transmission (including in neighbouring countries), is likely to be the single most important programmatic change for improving elimination prospects regionally.

\section{Strengths and limitations}

Surveillance quality affects the utility of evaluation tools to inform management. We considered surveillance quality to be sufficient throughout Mexico and Brazil based on assessment of indicators from the last 5 years including annual dog vaccinations in every state, adequate sample submissions and regular proficiency testing of laboratories [36], further supported by interviews with rabies programme managers and through PAHO missions. For other countries with less progressed control programmes and weaker surveillance, application of this tool would not be appropriate. For example, settings where locally-acquired human rabies cases are reported in the absence of confirmed animal cases would be indicative of inadequate surveillance. A particular concern is that the absence of detected cases reflects weak surveillance and not elimination. Although our requirement for a decline in case detection concomitant with dog vaccination prior to classification to putatively Absent guards against this, we recommend initial review to determine whether a country would benefit from this tool, for example, progression on SARE to at least step 3. Most countries in sub-Saharan Africa and Asia are probably at too early a stage in their control efforts for this tool to yet be of use, but we anticipate that in Latin America this tool could be applied to most countries and its use would complement SIRVERA.

Using sub-sampled simulated data, we demonstrated the extent to which our tool was robust to surveillance quality in endemic settings (Fig. 2). Surveillance had to reach very low levels or be very biased for states to be misclassified $(<2.5 \%$ detection in Endemic/Declining classifications). This threshold detection level increased to $5 \%$ as incursions become the main source of cases i.e. in Intermittent and Absent-Vulnerable classifications. We therefore emphasize the need to enhance surveillance to detect at least $5 \%$ of circulating cases, a recommendation consistent with prior work showing that $5 \%$ 


\section{Pathway to rabies elimination}

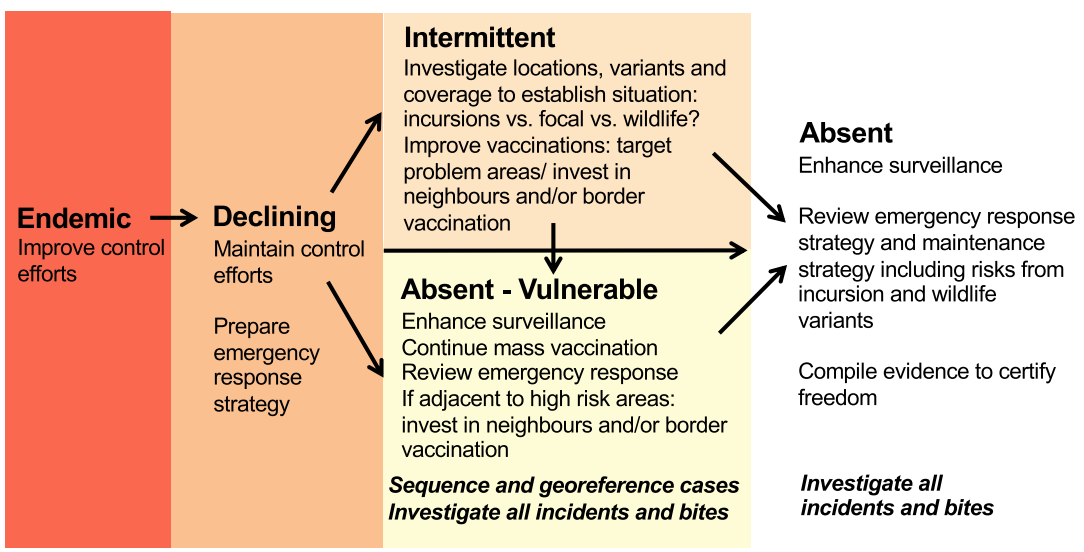

Fig. 4 Putative epidemiological classifications and associated management actions for progression towards elimination

case detection is required to verify disease freedom [9]. Integrated Bite Case Management shows promise as a tool that can detect $>10 \%$ of circulating cases [27], but further work is needed to assess its feasibility for implementation across a wide range of settings. We also emphasize that classification of Absent-Vulnerable and Absent using this tool is only putative and does not certify or guarantee rabies-free status. However, we consider that this classification would be a strong indication of readiness, when a country or state is well positioned to compile evidence of freedom in line with OIE requirements [11].

We recommend applying this tool over a five-year window of surveillance data, as shorter periods tend to magnify transient patterns and longer windows are potentially less responsive to epidemiological transitions. Case data should be reported by state and municipality, as this also provides a simple criterion for assessing surveillance quality. In Mexico and Brazil, case locations were complete at state-level from 2011 onwards and therefore did not affect later classifications; however, classifications earlier in the study may have been affected by this missing information. Municipality information could also be useful as elimination is approached; in fact, we suggest that incorporating municipality information into inference approaches could generate a better understanding of incursion risks and more tailored management recommendations to prevent them. Future work testing the application of this tool in other countries and at other spatial scales would be valuable to better understand its utility.

\section{Surveillance implications}

With the progressive control and elimination of dogmediated rabies from the region, other circulating virus variants have become increasingly apparent [37-39]. Variant identification played a discriminatory role for our classifications of states in both Mexico and Brazil, where both dog- and wildlife-associated variants have co-occurred. Circulation of wildlife variants is not necessarily an obstacle to elimination of dog rabies variants, but wildlife variants have potential to spread in the dog population and pose public health risks. Strategies for maintaining rabies freedom and for judicious use of post-exposure prophylaxis therefore need to account for these potentially complex situations, which may affect the scaling back of dog vaccination and protocols for identifying and treating bite victims. We therefore recommend that as states approach elimination (Intermittent, Absent-Vulnerable or Absent classification), sequencing of all detected cases be undertaken (Fig. 4). Since only very few cases are detected in these situations, this should not be cost prohibitive, though, baseline characterization of historically circulating variants is required.

The target for both Mexico and Brazil is now nationwide interruption of transmission and verification of disease freedom. For diseases that have been eradicated or regionally eliminated, intensified surveillance approaches have been employed to increase case detection [40-42]. Such approaches are now urgently required for areas classified as Absent or Absent-Vulnerable, or with Intermittent detection, to resolve uncertainties regarding viral circulation, initiate early outbreak responses and verify freedom (Table 4). We suggest that surveillance guided by Integrated Bite Case Management [43], with epidemiological investigations triggered by bites from suspicious animals, should enable verification of rabies freedom and guide scaling back of mass dog vaccination [27]. This approach should also help to identify 
Table 4 Management guidance based on classifications

\begin{tabular}{|c|}
\hline $\begin{array}{l}\text { onstrably } \\
\text { ined. Plans } \\
\text { ing } \\
\text { d } \\
\text { i) } \\
\text { ion has } \\
\text { zulating } \\
\text { ormation is } \\
\text { variants } \\
\text { ursions to } \\
\text { erwise } \\
\text { ion coverage } \\
\text { ion and in }\end{array}$ \\
\hline
\end{tabular}

transmission of other rabies variants and ensure appropriate treatment for exposed persons [44]. Moreover, this approach should result in sample submissions, as well as observation or quarantining of biting animals [45] which are important surveillance indicators. Likewise, genomic surveillance is a valuable tool [46], that used in combination with epidemiological data, could be crucial in guiding the rabies endgame. Sequencing of viruses can resolve key questions about viral circulation, discriminating wildlife variants from dog variants [37], including the potential for host shifts [47], and providing insights about the persistent lineages in remaining foci [48], which would be useful now in Chiapas state, Mexico and in Guatemala. In a previously rabies-free area, sequencing could identify the source(s) of incursions [49], and confirm that the new virus lineage differed from those historically circulating (i.e. undetected endemic circulation) [30, 50]. Adding functionality within SIRVERA for mapping georeferenced sequence data could facilitate rapid assessment of circulating viral lineages and potential incursion threats. Finally, contingency plans are urgently needed for states approaching elimination, and these should be regularly reviewed to ensure response capacity is maintained (Fig. $4)$.

\section{Conclusion}

The management tool we have developed adds to an increasing toolbox available to rabies managers to support them in determining their progress towards elimination, while providing tailored guidance for different epidemiological situations based on objective criteria derived from routine surveillance data. The added value of this tool is the direct use of surveillance data to provide quantitative measures of progress, but as a consequence this tool can only be applied to states with established surveillance capacity. The resulting management recommendations from applying this tool are logical and may appear selfevident (Fig. 4). But in practice, budget and human resource for post-vaccination monitoring and surveillance activities are limited, and often are not undertaken unless a strong case can be made as to their importance. This tool provides this critical evidence in a geographically targeted way. In summary, we identified the key remaining challenges to elimination of dog-mediated rabies from Mexico and Brazil as: 1) interruption of transmission from focally persistent states/borders that pose wider incursion risks; and 2) enhancing surveillance to distinguish variants, respond to and minimize incursion risks and verify freedom from disease, in order to allow relaxation of control measures without risking reemergence. Application of this management tool in Latin America could be used to prioritize efforts to accelerate progress towards regional elimination and ensure readiness for verification and maintenance of rabies freedom. Incorporation of this tool into the SIRVERA platform would encourage further engagement and use by programme managers. More broadly, we suggest this tool to be adapted and used effectively in other parts of the world to guide progress towards the global elimination of dog-mediated rabies.

\section{Supplementary information}

Supplementary information accompanies this paper at https://doi.org/10. 1186/s12879-020-05457-x.

\section{Additional file 1. \\ Additional file 2. \\ Additional file 3 . \\ Additional file 4}

Additional file 5 .

\section{Abbreviations}

PAHO: the Pan American Health Organization; SIRVERA: the Regional Epidemiologic Surveillance System for Rabies; SARE: Stepwise Approach towards Rabies Elimination 


\section{Acknowledgements}

This work was possible as a result of an enormous effort by government personnel across Latin America implementing rabies control programmes and collecting surveillance data over several decades and the support and dedication of Panaftosa and PAHO. We are especially grateful to Mary Freire de Carvalho, Ottorino Cosivi, Júlio Pompei, Felipe Rocha and colleagues from PAHO who facilitated and supported this work, Richard Reeve for help with funding acquisition and Shiny App development, Jamie Owen for development of the Shiny App and colleagues at BAHCM for useful feedback and comments on the manuscript.

\section{Authors' contributions}

$\mathrm{KR}, \mathrm{VDRV}$ and $\mathrm{KH}$ designed the study and KR, RM and $\mathrm{KH}$ carried out the analysis. STMB, EC, AC, VG, LRM, SR, MV and JFGR contributed data and VDRV oversaw the collection and collating of the data. KR and $\mathrm{KH}$ drafted the manuscript and STMB, DTH, PJ, RM, VDRV critically reviewed and revised the manuscript. All authors contributed to the interpretation of data and read and approved the final manuscript.

\section{Funding}

This research was supported by the Wellcome Trust (095787/Z/11/Z and 207569/Z/17/Z) and the BBSRC (BB/GCRF-IAA/08 and BB/M01116X/1). The funders had no role in in the design of the study, the collection, analysis and interpretation of data, or in writing the manuscript.

\section{Availability of data and materials}

The datasets (figures and code) generated and/or analysed during the current study are available in a private GitHub repository, and available from the corresponding author on reasonable request.

\section{Ethics approval and consent to participate}

Not applicable.

\section{Consent for publication}

Not applicable.

\section{Competing interests}

The authors declare that they have no competing interests.

\section{Author details}

${ }^{1}$ University of Warwick, School of Life Sciences, Gibbet Hill Road, Coventry, UK. ${ }^{2}$ University of Glasgow, Institute of Biodiversity, Animal Health and Comparative Medicine, Graham Kerr building, MVLS, Glasgow G12 8QQ, UK. ${ }^{3}$ Pan American Health Organization (PAHO), Duque de Caxias, Rio de Janeiro, Brazil. ${ }^{4}$ Virology, Central Laboratory, State Center for Health Surveillance, State Department of Health, São Paulo, Rio Grande do Sul, Brazil. ${ }^{5}$ Ministry of Health, Brasília, Distrito Federal, Brazil. ${ }^{6}$ Ministry of Health, Mexico, Distrito Federal, Mexico. ${ }^{7}$ University of Surrey, School of Veterinary Medicine, VSM Building, Guildford, UK.

Received: 5 August 2019 Accepted: 27 September 2020

Published online: 20 October 2020

\section{References}

1. Hampson K, Coudeville L, Lembo T, Sambo M, Kieffer A, Attlan M, et al. Estimating the global burden of endemic canine rabies. PLoS Negl Trop Dis. 2015;9(4):e0003786.

2. Abela-Ridder B, Knopf L, Martin S, Taylor L, Torres G, de Balogh K. 2016: the beginning of the end of rabies? Lancet Glob Health. 2016;4(11):e780-1. https://doi.org/10.1016/S2214-109X(16)30245-5.

3. Vigilato MA, Clavijo A, Knobl T, Tamayo Silva HM, Cosivi O, Schneider MC, et al. Progress towards eliminating canine rabies: policies and perspectives from Latin America and the Caribbean. Philos Trans R Soc Lond B Biol Sci. 2013;368(1623):20120143.

4. WHO. The sixth meeting of the International Coordinating Group (ICG) of the Bill \& Melinda Gates foundation-World Health Organization project on eliminating human and dog rabies. Durban, South Africa; 2014.

5. OIE. ASEAN Rabies Elimination Strategy. World Organisation for Animal Health; 2015.
6. Minghui R, Stone M, Semedo MH, Nel L. New global strategic plan to eliminate dog-mediated rabies by 2030. Lancet Glob Health. 2018;6(8):e8289. https://doi.org/10.1016/S2214-109X(18)30302-4.

7. Rist CL, Arriola CS, Rubin C. Prioritizing Zoonoses: a proposed one health tool for collaborative decision-making. PLoS One. 2014;9(10):e109986.

8. Munyua P, Bitek A, Osoro E, Pieracci EG, Muema J, Mwatondo A, et al. Prioritization of zoonotic diseases in Kenya, 2015. PLoS One. 2016;11(8): e0161576.

9. Wallace RM, Undurraga EA, Blanton JD, Cleaton J, Franka R. Elimination of Dog-Mediated Human Rabies Deaths by 2030: Needs Assessment and Alternatives for Progress Based on Dog Vaccination. Front Vet Sci. 2017;4:9.

10. WHO. Expert Consultation on Rabies, Third Report. Geneva: WHO; 2018.

11. World Organization for Animal health. Rabies (infection with Rabies Virus). Manual of Diagnostics Tests and Vaccines for Terrestrial Animals: http://www. oie.int/fileadmin/Home/eng/Health_standards/tahm/2.01.17_RABIES.pdf; 2017.

12. Lembo T. Partners for Rabies Prevention. The Blueprint for Rabies Prevention and Control: A Novel Operational Toolkit for Rabies Elimination. Plos Neglect Trop Dis. 2012;6(2):e1388.

13. Octaria R, Salyer SJ, Blanton J, Pieracci EG, Munyua P, Millien M, et al. From recognition to action: a strategic approach to foster sustainable collaborations for rabies elimination. PLoS Negl Trop Dis. 2018;12(10): e0006756.

14. OIE/ WHO /FAO/ The Global Alliance for Rabies Control. The Stepwise Approach towards Rabies Elimination. https://caninerabiesblueprint.org/Astepwise-approach-to-planning. [cited 28 Sept 2020].

15. Del Rio Vilas VJ, Freire de Carvalho MJ, MAN V, Rocha F, Vokaty A, Pompei JA, et al. Tribulations of the Last Mile: Sides from a Regional Program. Front Vet Sci. 2017;4(4). https://doi.org/10.3389/fvets.2017.00004.

16. REDIPRA. Meeting of Rabies Programme Directors of the Americas - Report Recommendations. http://www.panaftosa.org/redipra15/index.php?option= com_content\&view=article\&id=81\&ltemid=78\&lang=en; 2015.

17. Pan American Health Organization. Epidemiological Alert [Internet] Jun Available: http://www.paho.org/hq/index.php?option=com_docman\&task= doc_download\&ltemid=\&gid=30659\&lang=en. 2015.

18. Freire de Carvalho M, MAN V, Pompei JA, Rocha F, Vokaty A, Molina-Flores B, et al. Rabies in the Americas: 1998-2014. PLOS Neglect Trop Dis. 2018; 12(3):e0006271.

19. Velasco-Villa A, Mauldin MR, Shi M, Escobar LE, Gallardo-Romero NF, Damon I, et al. The history of rabies in the Western hemisphere. Antivir Res. 2017; 146:221-32.

20. Townsend SE, Lembo T, Cleaveland S, Meslin FX, Miranda ME, Putra AAG, et al. Surveillance guidelines for disease elimination: a case study of canine rabies. Comp Immunol Microbiol Infect Dis. 2013;36(3):249-61.

21. Townsend SE, Sumantra IP, Pudjiatmoko BGN, Brum E, Cleaveland S, et al. Designing Programs for Eliminating Canine Rabies from Islands: Bali, Indonesia as a Case Study. Plos Neglect Trop Dis. 2013;7(8):e2372.

22. Hampson K, Dushoff J, Cleaveland S, Haydon DT, Kaare M, Packer C, et al. Transmission dynamics and prospects for the elimination of canine rabies. PLoS Biol. 2009;7(3):e1000053.

23. Davlin SL, VonVille HM. Canine rabies vaccination and domestic dog population characteristics in the developing world: A systematic review. Vaccine. 2012;30(24):3492-502.

24. Rangel MCF, Cardenas Lara J, DeAlujy AS. The canine population of Mexico City: an estimativ study. Anim Reg Stud. 1981;3:281-90.

25. Garcia RCM, Amaku M, Biondo AW, Ferreira F. Dog and cat population dynamics in an urban area: evaluation of a birth control strategy. Pesquisa Veterinária Brasileira. 2018;38:511-8.

26. Ferguson EA, Hampson K, Cleaveland S, Consunji R, Deray R, Friar J, et al. Heterogeneity in the spread and control of infectious disease: consequences for the elimination of canine rabies. Sci Rep. 2015;5:18232. https://doi.org/10.1038/srep18232.

27. Hampson K, Abela-Ridder B, Brunker K, Bucheli STM, Carvalho M, Caldas E, Changalucha J, Cleaveland S, Dushoff J, Gutirrez V, Fooks AR, Hotopp K, Haydon DT, Lugelo A, Luhasi K, Mancy R, Marston DA, Mtema Z, Rajeev M, Montebello P, Dourado LR, Gonzalez Roldan JF, Rysava K, Rocha SM, Sambo M, Sikana L, Vigilato M, Del Rio Vila V. Surveillance to establish Elimination of Transmission and Freedom from Dog-mediated Rabies. BioRxiv. 2016. https://www.biorxiv.org/content/10.1101/096883v1.full.

28. WHO, FAO, OIE, GARC. Zero by 30: The global strategic plan to end human deaths from dog-mediated rabies by 2030. http://www.who.int/rabies/ United_against_Rabies/en/ 2018. 
29. Office International des Epizooties. Chapter 8.13, Infection with Rabies Virus. Paris: Terrestrial Animal Health Code; 2016.

30. Bourhy H, Nakoune E, Hall M, Nouvellet P, Lepelletier A, Talbi C, et al. Revealing the Micro-scale Signature of Endemic Zoonotic Disease Transmission in an African Urban Setting. Plos Pathogens. 2016;12(4): e1005525.

31. Beyer HL, Hampson K, Lembo T, Cleaveland S, Kaare M, Haydon DT. Metapopulation dynamics of rabies and the efficacy of vaccination. Proceed R Soc B Biol Sci. 2011;278(1715):2182-90.

32. Castillo-Neyra R, Brown J, Borrini K, Arevalo C, Levy MZ, Buttenheim A, et al. Barriers to dog rabies vaccination during an urban rabies outbreak: Qualitative findings from Arequipa, Peru. PLOS Neglect Trop Dis. 2017;11(3): e0005460.

33. Tohma K, Saito M, Demetria CS, Manalo DL, Quiambao BP, Kamigaki T, et al. Molecular and mathematical modeling analyses of inter-island transmission of rabies into a previously rabies-free island in the Philippines. Infect Genet Evol. 2016:38:22-8

34. Sambo M, Hampson K, Changalucha J, Cleaveland S, Lembo T, Lushasi K, et al. Estimating the size of dog populations in Tanzania to inform rabies control. Veterinary Sciences. 2018;5(3):77.

35. Sambo M, PCD J, Hotopp K, Changalucha J, Cleaveland S, Kazwala R, et al. Comparing Methods of Assessing Dog Rabies Vaccination Coverage in Rural and Urban Communities in Tanzania. Front Vet Sci. 2017;4(33). https://doi. org/10.3389/fvets.2017.00033.

36. Clavijo A, Freire de Carvalho MH, Orciari LA, Velasco-Villa A, Ellison JA, Greenberg $L$, et al. An inter- laboratory proficiency testing exercise for rabies diagnosis in Latin America and the Caribbean. PLOS Neglect Trop Dis. 2017; 11(4):e0005427.

37. Carnieli P, Fahl WD, Castilho JG, Oliveira RD, Macedo Cl, Durymanova E, et al. Characterization of rabies virus isolated from canids and identification of the main wild canid host in northeastern Brazil. Virus Res. 2008;131(1):3346.

38. Favoretto SR, de Mattos CC, Morais NB, Alves Araujo FA, de Mattos CA. Rabies in marmosets (Callithrix jacchus), Ceara, Brazil. Emerg Infect Dis. 2001; 7(6):1062-5

39. Rocha SM, de Oliveira SV, Heinemann MB, Gonçalves VS. Epidemiological Profile of Wild Rabies in Brazil (2002-2012). Transbound Emerg Dis. 2015; 64(2):624-33.

40. Mariner JC, House JA, Mebus CA, Sollod AE, Chibeu D, Jones BA, et al. Rinderpest eradication: appropriate technology and social innovations. Science. 2013;337(6100):1309-12.

41. Blake IM, Burton MJ, Bailey RL, Solomon AW, West S, Munoz B, et al. Estimating Household and Community Transmission of Ocular Chlamydia trachomatis. Plos Neglect Trop Dis. 2009;3(3):e401.

42. Foege WH, Millar JD, Lane JM. Selective epidemiologic control in smallpox eradication. Am J Epidemiol. 1971;94(4):311-5.

43. Undurraga EA, Meltzer MI, Tran CH, Atkins CY, Etheart MD, Millien MF, et al. Cost-effectiveness evaluation of a novel integrated bite case management program for the control of human rabies, Haiti 2014-2015. Am J Trop Med Hyg. 2017:96(6):1307-17.

44. Etheart MD, Kligerman M, Augustin PD, Blanton JD, Monroe B, Fleurinord L, et al. Effect of counselling on health-care-seeking behaviours and rabies vaccination adherence after dog bites in Haiti, 2014-15: a retrospective follow-up survey. Lancet Glob Health. 2017;5(10):E1017-E25.

45. Medley AM, Millien MF, Blanton J, Ma X, Augustin PD, Crowdis K, et al. Retrospective Cohort Study to Assess the Risk of Rabies in Biting Dogs, 2013-2015, Republic of Haiti. Trop Med Infect Dis. 2017;2(2):14. https://doi. org/10.3390/tropicalmed2020014.

46. Gardy JL, Loman NJ. Towards a genomics-informed, real-time, global pathogen surveillance system. Nat Rev Genet. 2018;19:9-20.

47. Mollentze N, Biek R, Streicker DG. The role of viral evolution in rabies host shifts and emergence. Curr Opin Virol. 2014;8:68-72.

48. Brunker K, Marston DA, Horton DL, Cleaveland S, Fooks AR, Kazwala R, Ngeleja C, Lembo T, Sambo M, Mtema ZJ, Sikana L, Wilkie G, Biek R, Hampson K. Elucidating the phylodynamics of endemic rabies virus in eastern Africa using whole-genome sequencing. Virus Evol. 2015;1(1): vev011. https://doi.org/10.1093/ve/vev011

49. Mollentze N, Weyer J, Markotter W, le Roux K, Nel LH. Dog rabies in southern Africa: regional surveillance and phylogeographical analyses are an important component of control and elimination strategies. Virus Genes. 2013;47(3):569-73.
50. Zinsstag J, Lechenne M, Laager M, Mindekem R, Naissengar S, Oussiguere A, et al. Vaccination of dogs in an African city interrupts rabies transmission and reduces human exposure. Sci Transl Med. 2017;9(421):eaaf6984. https:// doi.org/10.1126/scitranslmed.aaf6984.

\section{Publisher's Note}

Springer Nature remains neutral with regard to jurisdictional claims in published maps and institutional affiliations.
Ready to submit your research? Choose BMC and benefit from:

- fast, convenient online submission

- thorough peer review by experienced researchers in your field

- rapid publication on acceptance

- support for research data, including large and complex data types

- gold Open Access which fosters wider collaboration and increased citations

- maximum visibility for your research: over $100 \mathrm{M}$ website views per year

At BMC, research is always in progress.

Learn more biomedcentral.com/submissions 